Meta

Journal des tradlucteurs

Translators' Journal

\title{
Pour une méthode générale de traduction : traduire par l'interprétation du texte
}

\section{Jean-Claude Gémar}

Volume 35, numéro 4, décembre 1990

URI : https://id.erudit.org/iderudit/002853ar

DOI : https://doi.org/10.7202/002853ar

Aller au sommaire du numéro

Éditeur(s)

Les Presses de l'Université de Montréal

ISSN

0026-0452 (imprimé)

1492-1421 (numérique)

Découvrir la revue

Citer cet article

Gémar, J.-C. (1990). Pour une méthode générale de traduction : traduire par

l'interprétation du texte. Meta, 35(4), 657-668. https://doi.org/10.7202/002853ar d'utilisation que vous pouvez consulter en ligne.

https://apropos.erudit.org/fr/usagers/politique-dutilisation/ 


\section{POUR UNE MÉTHODE GÉNÉRALE DE TRADUCTION: TRADUIRE PAR L'INTERPRÉTATION DU TEXTE*}

JEAN-CLAUDE GÉMAR

Université de Montréal, Montréal, Canada

Le présent travail découle d'un ensemble de constats portant sur certaines lacunes relevées chez les traducteurs de la nouvelle génération et les étudiants se destinant à la traduction. Ces carences sont régulièrement dénoncées par les employeurs, les professeurs de traduction et les traducteurs appartenant à la génération précédente. Elles caractérisent un phénomène particulièrement répandu - et également dénoncé - dans les sociétés industrielles avancées du monde occidental.

Ce sont, parmi les plus importantes et compte non tenu du problème que pose l'orthographe, la faiblesse de la «culture générale» du traducteur, son absence d'autonomie et sa connaissance des langues, la maternelle y comprise.

Des trois, je dirais que la plus grave me semble être l'absence d'autonomie, car elle révèle un défaut de «structure», une faille dans l'intellect et un manque de confiance en soi; d'où le recours incessant à des aides tels que les dictionnaires pour y chercher la signification des mots - dans le cas d'une langue étrangère - leur orthographe et l'explication des choses.

Ensuite, les faiblesses patentes de la culture générale du traducteur indiquent une méconnaissance coupable du monde, de la société et de l'environnement dans lequel il vit. Par culture générale je n'entends pas seulement la culture «littéraire», mais aussi et surtout la connaissance des faits, événements, progrès et autres réalités, indispensable chez le traducteur, à défaut de quoi il ne saisira pas dans toute son étendue le sens réel, profond, des textes qu'il doit traduire.

Quant à la connaissance déficiente des langues, maternelle et seconde, il s'ensuit que le traducteur comprend insuffisamment le texte de départ et que ce texte, dont le message est tronqué, sera mal exprimé et péchera aussi par la forme.

Le professeur de traduction désireux de remédier à cette situation et d'aider ses élèves devra s'attacher à combler ces trois lacunes graves. La difficulté sera variable selon qu'il cherchera à développer l'autonomie des étudiants, à étendre ou renforcer leur culture générale ou à perfectionner leurs connaissances linguistiques. Les situations particulières, en outre, rendront sa tâche plus ou moins aisée.

Mon propos se bornera à présenter en quoi consiste la méthode que je préconise, une fois que j'en aurai justifié le besoin et que je me serai expliqué sur le cheminement intellectuel qui m'a conduit à parler d'une méthode de formation du traducteur par l'interprétation du texte.

\section{LA FINALITÉ D'UNE MÉTHODE DE TRADUCTION PAR L'INTERPRÉTATION DU TEXTE}

Par cette méthode, je vise deux objectifs principaux:

1. Former des traducteurs plus autonomes, plus complets, et donc mieux préparés à faire face aux responsabilités croissantes que leur confient les employeurs ou qui les attendent dans le service de traduction de demain, ou encore qu'exige d'eux une société en évolution rapide.

Meta, XXXV, 4, 1990 
2. Pour ce faire, introduire dans la formation - à un niveau avancé — du traducteur le fruit des réflexions, constats et travaux réalisés tant en traductologie que dans les disciplines complémentaires ou périphériques.

Cela dit, je ne nie pas les mérites des nombreuses et excellentes méthodes de traduction que nous connaissons et utilisons tous plus ou moins. Il me semble toutefois que celles-ci ne voient dans le traducteur ou l'étudiant en traduction qu'un «être professionnel» et non un être humain d'abord et avant tout. On fait semblant de croire que l'on ne formera que des traducteurs, alors que cette formation ne constitue qu'une étape dans la carrière, le cheminement personnel et, finalement, la vie d'une personne. L'être professionnel n'est pas désincarné, et pour faire des traducteurs sereins, épanouis et en pleine possession de leurs moyens, il importe qu'ils aient la «tête bien faite» avant de l'avoir «bien pleine».

Je souhaite que l'on cesse de réduire la traduction à un acte isolé ou «gratuit», que l'on arrête de la considérer comme une opération relevant, pour les uns, uniquement de la langue, et, pour les autres, seulement de la parole, et qu'elle soit enfin analysée comme l'activité sinon la discipline véritablement intégrée qu'elle devrait être. Autrement dit, à partir des constatations et observations inspirées par la pratique de l'activité traduisante, dégager les constats théoriques et méthodologiques que celle-ci impose, et non le contraire (soit partir d'une base théorique pour en construire une pratique).

Je propose ici que l'on inverse la pyramide et que de la base - la pratique - on remonte vers le sommet, dans un mouvement d'interaction incessante, au lieu de partir du sommet pour dicter à la base une conduite conçue et établie de façon arbitraire et artificielle. Cette dernière approche est totalement déconnectée de la réalité de la traduction, qui n'est nullement un acte unilatéral de la part du traducteur, mais plutôt dialogal puisqu'il construit son texte au fil d'un dialogue, incessant quoique muet, entre l'auteur du texte de départ et lui-même. L'interprétation finale - et personnelle - que fait le traducteur lui est, à l'évidence, entièrement imputable, mais n'en est pas moins le produit de la (re)construction du sens à laquelle il arrive en extrayant ses réponses du tréfonds du texte à traduire, à coups d'interrogations lancées à l'auteur invisible et néanmoins présent.

\section{LE CHEMINEMENT INTELLECTUEL}

Je suis parti de trois constats. Le premier se fonde sur une réflexion de jurilinguiste, le deuxième sur celle du traductologue, et le troisième, sur une intime conviction.

\section{LAPPORT DE LA JURILINGUISTIQUE}

Depuis l'apparition de l'homo scribens, l'habitude est prise de chercher à percer, à approfondir, à (re)trouver le sens caché (= véritable, profond, premier - David, 1980) des textes produits, pour diverses raisons.

Aussi, pour parvenir à leurs fins, juristes, religieux, philologues et autres glossateurs invétérés ont-ils pris l'habitude de faire, chacun avec ses méthodes et en fonction des objectifs particuliers visés, l'interprétation des textes, normatifs ou non, qu'ils sont appelés à élucider. Quelles que soient les méthodes d'interprétation utilisées, souvent fort différentes les unes des autres, le but visé reste le même, en gros, que celui du traducteur: (re)trouver le sens d'un texte.

Là où les démarches des premiers diffêrent de celles du traducteur, c'est sur la finalité de l'acte d'interprétation. De toute évidence, les desseins du législateur, du commentateur de la Bible, du philologue ou du philosophe ne sont pas du même ordre que ceux du traducteur, lequel, de surcroît, travaille toujours à partir d'une — ou vers une - langue étrangère. 
Les préoccupations du jurilinguiste, toutefois, rejoignent celles du traducteur : 1'un comme l'autre cherchent à produire un texte - loi, règlement ou traduction - final de qualité, sur le double plan du contenu et de la forme.

\section{L'APPORT DE LA TRADUCTOLOGIE}

Qui pourrait nier que la traductologie, à l'instar de la linguistique pure, s'intéresse davantage, depuis une trentaine d'années, aux questions de «langue» qu'aux productions de la «parole»? Le souci de formalisme, les recherches expérimentales en laboratoire n'expliquent pas tout.

Les traductologues, sans doute sous l'influence des linguistes - notamment des structuralistes et des générativistes -, ont délibérément ignoré le fait que la traduction, qu'elle soit envisagée comme texte écrit ou oral, est avant tout une pratique, et une pratique humaine, donc vivante et dynamique - de façon consciente ou non - autant que statique. En clair, la traduction fait intervenir un élément essentiel, généralement exclu par les chercheurs parce qu'il est rebelle à l'observation et à l'analyse «scientifiques»: le processus mental.

Or, continuer à refuser de prendre en compte le rôle que joue l'esprit - le cerveau humain - dans l'opération de traduction revient à nier les progrès indéniables accomplis dans la connaissance de mécanismes qui échappaient traditionnellement à l'observation et à l'analyse. Les travaux des neurologues et neurobiologistes, des psycho- et neurolinguistes, des biologistes et des psychologues renforcent ma conviction que l'acquis joue un rôle prépondérant dans le fonctionnement et la «performance» de l'esprit.

La traductologie ne peut que bénéficier de ces recherches. Elle doit s'ouvrir davantage aux disciplines susceptibles de nourrir la réflexion des traductologues si l'on veut qu'elle progresse dans l'affirmation de plus en plus fondée de son objet - expliciter la traduction - et le raffinement de ses méthodes.

\section{LE TRADUCTEUR, ÊTRE DIALOGAL}

J'ai l'intime conviction que l'acte de traduction constitue un acte dialogal, quoique solitaire et isolé, et même muet, tout en étant une opération éminemment individuelle (après tout, c'est le traducteur qui décide, en fin de compte, de retenir telle interprétation du texte plutôt que telle autre, en fonction de son bagage personnel et de l'intelligence qu'il a de la lettre et de l'esprit du texte). Et, de fait, il y a dialogue entre le traducteur et le texte de départ et, par delà celui-ci, l'auteur du texte, lequel peut être un collectif comme dans le cas d'une loi - ou un individu — prenons le cas d'un testament, d'une lettre, d'un poème, etc.

Cette relation dialogale, cependant, ne doit pas être comprise dans le sens courant, «vivant», du terme, mais plutôt comme un «dialogue intérieur» au cours duquel le traducteur interroge à la fois le texte/auteur et son propre fonds de connaissances auquel il confronte les réponses obtenues du premier. C'est au terme de ce double dialogue, incessant et ultrarapide, que le traducteur retiendra l'interprétation à laquelle son analyse l'aura conduit.

Ces trois constats doivent nous faire réfléchir sur nos méthodes d'enseignement de la traduction. La somme de leur addition m'incline à penser que nous devrions commencer sans plus tarder à nous interroger sur notre pédagogie de la traduction et ses grandes orientations, en somme, sur sa finalité et son objet.

\section{TRADUIRE PAR L'INTERPRÉTATION DU TEXTE :}

\section{MÉTHODE OU PÉDAGOGIE DE LA TRADUCTION ?}

Le monde de la traduction possède déjà de nombreuses méthodes, «recettes» et formules largement répandues et exploitées. Il est donc tout à fait légitime de se poser la 
question suivante: que peut attendre le traducteur d'une nouvelle méthode? Que peut-elle apporter à une profession détenant ses lettres de noblesse, à un milieu saturé de conseils sur la «manière de traduire»? En d'autres termes, quelle est son utilité, sa justification?

Au terme d'une observation attentive et continue de l'activité traduisante, à titre de professeur de traduction et d'observateur passionné par les faits de langue, je crois pouvoir dire que les traductologues ou didacticiens de la traduction sont encore loin d'avoir tiré tout le parti possible des immenses ressources que recèle le processus mental mis en jeu par l'acte de traduction. Peu d'entre nous se sont en outre plongés dans le vaste corps doctrinal édifié par les juristes depuis qu'ils s'interrogent sur l'interprétation à donner au langage du droit, soit depuis des milliers d'années.

C'est pour tenter de combler une partie de cette lacune que j'ai entrepris de prospecter un champ d'investigation aussi riche de promesses. Ma recherche visait principalement à établir les principes d'une méthode de formation des traducteurs qui réponde aux besoins multiples de la société actuelle. Je me proposais, par la même occasion, de répondre aussi à ceux de la société de demain que l'on peut induire des prévisions, tendances et orientations générales caractérisant son évolution, en particulier sur les plans économique, scientifique et technique, social et juridique.

\section{L'HYPOTHÈSE DE BASE}

Je suis parti d'une constatation simple: le traducteur, aux prises avec son texte, ne dispose généralement pas d'autres ressources que son intelligence et son jugement pour trouver la solution aux problèmes qu'il est appelé à résoudre. Une fois qu'il aura lu - et relu - son texte, il procédera aux analyses requises, donc logique, grammaticale et terminologique. Lorsqu'il aura effectué celles-ci (cf. Delisle, 1980), il lui restera à saisir le sens des divers éléments du message.

Ici intervient l'hypothèse que le traducteur est alors amené à interpréter — au sens commun du terme: (re)donner/(re)trouver un sens à - le texte selon un processus mental complexe durant lequel s'engage un dialogue intérieur sous forme de questions et de réponses qui conduira au transcodage en langue d'arrivée des données du message contenu dans le texte rédigé dans la langue de départ. Je postule que le succès de l'opération dépendra pour une bonne part de la «banque de données» emmagasinées dans le cerveau du traducteur, autrement dit de son bagage, de ses connaissances, et de sa capacité à en tirer un parti optimal, soit de son savoir-faire.

\section{LES DONNÉES THÉORIQUES}

La recherche que j'ai entreprise est placée au carrefour des voies actuelles de la connaissance parce que la traduction est au cœur du flot croissant de la «communication», phénomène qui caractérise, volens nolens, notre époque.

Cette recherche fait intervenir, d'une part, la conception empirique de la réalité par la mise en œuvre de données découlant de l'expérimentation, de faits observables et observés et, d'autre part, une conception holistique du réel où la matière (le corps humain) et l'immatériel (l'esprit humain) sont en interdépendance (cf. Prigogine, 1979), autant que peuvent l'être la forme et le contenu d'un énoncé (Chomsky, 1965, 1980). Art, technique ou science, selon le regard qu'on lui accorde, la traduction est, à tout le moins, une discipline située à l'intersection de ces deux voies.

Je postule que le traducteur, dans l'acte d'interprétation sémantique du texte, fait intervenir tour à tour l'esprit de géométrie et l'esprit de finesse pascaliens, parfois les deux ensemble, qu'il met au service de son art - lorsqu'il s'élève à cette hauteur.

Les mécanismes de cette opération pourraient être mieux compris si les traductologues puisaient davantage dans le fonds quasi illimité du capital de connaissances accumulé, entre autres, par les philosophes, psychologues, biologistes, linguistes, informaticiens et 
juristes. Je crois qu'une telle «alliance» (au sens que lui donne Progogine) devrait favoriser une meilleure compréhension de «l'une des plus complexes opérations cognitivolinguistiques qu'il nous soit donné d'observer chez l'être humain» (Nespoulous, 1984: 8).

Les recherches actuelles en psycholinguistique et en neurolinguistique démontrent le rôle cardinal de l'interprétation du sens par le traducteur (Bouton, 1984). Celui-ci, en raison du caractère individuel de sa démarche et de la «lecture» personnelle qu'il fait du texte, peut faire une interprétation et dégager un sens différents de ce qu'envisageait l'auteur. Aussi est-il très difficile, voire, à la limite, presque impossible de garantir que les valeurs signifiées en A seront identiques en B. De plus, les «stratégies de traduction» (Le Dorze et Nespoulous, 1984) variant considérablement d'un traducteur à l'autre, toute tentative d'appréhension du phénomène de la traduction, d'explicitation de ses mécanismes et, a fortiori, toute velléité de systématisation constituent autant d'entreprises aléatoires et spéculatives. Enfin, pour compliquer le tout, il est même possible de traduire sans comprendre puisque la traduction peut être, dans certains cas, "neurofonctionnellement indépendante de la capacité de comprendre et de parler deux langues» (Paradis, 1984)!

\section{LE TRADUCTEUR EST-IL UN ETRE DE «RAISON»?}

De récents travaux en neurologie et en neurobiologie (Changeux, 1983), particulièrement en aphasie des bilingues (Paradis et Lecours, 1984), renforcent l'hypothèse que le traducteur, à l'instar de tout humain, procède davantage par interprétation personnelle, par «intuition», plutôt que par la voie du seul raisonnement, pour (re)trouver le sens du message contenu dans le texte de départ (TD) et le rendre dans le texte d'arrivée (TA).

Certains biologistes et généticiens parmi les plus éminents (Jacob, 1970 et 1981; Jacquard, 1978, 1982 et 1986) contestent fortement l'image réductrice de l'intelligence que la pédagogie, l'école et les tests psychotechniques ont contribué à façonner. D'ailleurs, qu'est-ce que l'intelligence? Et, si tant est qu'existe une certaine «intelligence», perçue comme aptitude, faculté supérieure qui distingue traditionnellement l'humain de l'animal, est-elle essentiellement acquise ou innée? Le débat est loin d'être tranché, quoique nombre de psychologues, dont Piaget (1970), et de biologistes penchent plutôt en faveur de l'acquis. Ce débat, on le retrouve en linguistique, ce qui a des répercussions sur la traductologie. Pour certains linguistes (Hagège, 1985: 63), les propriétés d'une langue seraient acquises; pour d'autres, plus formalistes (Chomsky, 1965: 201), elles seraient innées.

Des chercheurs réfléchissant sur l'intelligence artificielle (Waldrop, 1984) ou sur la langue technique (Thomas, 1982) contribuent à renforcer l'impression que l'acquis joue un rôle prépondérant dans le fonctionnement et, surtout, la «performance» de l'esprit humain.

Des philosophes enfin, notamment ceux de «l'esprit», viennent également étayer cette hypothèse: l'acquisition des connaissances serait par essence un acte individuel (Polanyi, 1967; Searle, 1986). Nous n'attribuons un sens à ce que nous lisons qu'en fonction de notre bagage, proportionnellement aux connaissances que nous avons acquises. Il s'ensuit qu'une compétence plus ou moins grande permettra de reconstruire plus ou moins justement le sens. Une telle hypothèse est lourde de conséquences pour le traducteur.

\section{UNE PÉDAGOGIE DE LA TRADUCTION AXÉE SUR L'INTERPRÉTATION DU SENS}

Selon les conditions énoncées, le traducteur devrait posséder le bagage requis pour tendre vers ce but: appréhender le mieux possible les divers composants sémantiques du message. Cette obligation du traducteur lui est bien évidemment personnelle, et le professeur de traduction ne peut intervenir utilement dans ce processus que si la tête de l'élève 
est «bien faite», à défaut d'être «bien pleine». Il reste que l'acquisition ou le renforcement du bagage du traducteur, notamment sur les plans lexical et notionnel, devrait faire l'objet de soins particuliers de la part des professeurs. De là l'intérêt d'une méthode orientée vers l'apprentissage individuel.

Je suis parti des postulats de la linguistique saussurienne et de ses concepts clés pour rejoindre la conception chomskyenne des rapports entre la forme et le sens en matière de langue, sans toutefois adhérer au principe de la dichotomie langue/parole ou toute autre façon de l'exprimer. L'analyse du discours (Charaudeau, 1983) a également retenu mon attention parce qu'elle fait intervenir la communication et le message, qui sont au cœur de l'activité traduisante. Je ne vais pas, toutefois, jusqu'à affirmer que la traduction n'est qu'un acte de communication, principe au nom duquel l'intention implicite - et même explicite - de l'auteur est parfois délibérément ignorée au profit du seul «destinataire». Cette conception individualiste - que j'oppose à l'attitude universaliste — du rôle du traducteur, qui se substitue discrétionnairement à l'auteur, n'est pas la mienne:

Plus modestement, je crois plutôt que le traducteur est un médiateur entre le texte de départ et le destinataire du texte d'arrivée, qu'il soit désigné ou anonyme, dans lequel je reconnais la société tout entière, voire «l'humaine condition».

Quelques exemples illustreront mon propos.

\section{ILLUSTRATION}

En fouillant dans nos souvenirs ou dans notre pratique de traducteur, nous a-t-il été donné de voir deux personnes produire la même traduction, rigoureusement identique dans la forme comme dans la substance, du même texte? Je vais plus loin: une même personne traduisant le même texte à plusieurs reprises sur une période donnée produirat-elle deux fois une version identique à l'une de ses propres traductions? En théorie, cela semble possible. Mais, en pratique, cela ne s'est probablement jamais réalisé, du moins au mieux de ma connaissance. Je poursuis une expérience entreprise voici quelques années avec des étudiants: je donne les mêmes passages de plusieurs textes à traduire plusieurs fois sur une année à plusieurs groupes de volontaires. Je corrige ces traductions - sans les noter: il s'agit d'une expérience! — puis en analyse les résultats, les compare à d'autres groupes témoins, etc.

Il est possible d'en déduire que l'instantané, l'éphémère caractérisent l'opération qu'effectue le traducteur, laquelle constitue en outre un phénomène unique dans sa réalisation puisqu'elle ne se répète pas, au moins quant à la forme du texte d'arrivée, jamais exactement le même. En revanche, le contenu — le fond ou message: le sens - est toujours reproduit ou rendu, le plus souvent avec exactitude, par le traducteur.

De ce constat découlent certaines hypothèses. Tout d'abord, le sens (signifié) d'un message peut être dissocié de la forme (linguistique) sous laquelle il est exprimé - le signe ou signifiant - de la même façon que l'opération de représentation du message traduit en mots et phrases est double: «sémantique d'abord, puis formelle» (Le Dorze et Nespoulous, 1984: 79).

Cela confirmerait ce que pensent intuitivement les gens qui, comme moi, reçoivent du courrier de diverses régions du monde rédigé en différentes langues et qui, après en avoir pris connaissance, en gardent le contenu en mémoire, indépendamment de la langue utilisée par l'auteur. Il s'ensuit que, dans le cas de la lecture, le sens des mots d'un texte, présent de façon inconsciente - ou «tacite», selon Polanyi — dans la pensée (mémoire), est activé et rappelé instantanément à la lecture d'un texte, et la solution, le sens du message, des mots - à condition qu'ils fassent partie du lexique mental du lecteur/traducteur, bien entendu — s'impose immédiatement à ce dernier. 
Ensuite, les notions fondamentales de connaissances (le «bagage») et d'expérience du lecteur sont à prendre en compte. À cet égard, une expérience réalisée aux États-Unis par un groupe de psychologues et d'informaticiens (Larkin, 1980) mérite d'être relatée. Deux groupes de physiciens, l'un composé d'experts, l'autre de jeunes physiciens encore peu aguerris, doivent résoudre des problèmes de physique dans des conditions expérimentales. Les physiciens expérimentés retrouvent la solution grâce à une combinaison de connaissances, de mémoire et d'expérience, sans recourir apparemment au raisonnement. Les jeunes physiciens, au contraire, font appel en priorité au raisonnement.

Le traducteur ne procède pas autrement. Le traducteur inexpérimenté ou débutant parvient, au terme d'une analyse plus ou moins rapide et poussée selon les conditions dans lesquelles il travaille (en situation d'examen ou de concours, par exemple), à reconstruire le sens que son stock de connaissances lui permet d'appréhender. Je postule qu'en l'occurrence le traducteur interprète ce sens.

Le traducteur expérimenté, plus aguerri, retrouve généralement le sens plus ou moins instantanément, sans analyse ou presque, parce qu'il le détient déjà en mémoire. Il ne fera appel à l'analyse ou au raisonnement logique que pour résoudre le problème nouveau, inconnu de lui - de sa mémoire - que lui posera un texte dont le domaine et sa terminologie sont extérieurs à son champ d'activité ou échappent à sa compétence, et ne font alors pas partie de ses connaissances. Dans ces conditions, je postule encore que le traducteur recourt à l'interprétation du sens d'un message lorsque celui-ci est opaque à son intelligence. Dans le cas contraire, tel le physicien expérimenté, il parvient à la solution (retrouver le sens) à partir de la combinaison, plus ou moins harmonieuse et efficace, de ses connaissances, de sa mémoire et de son expérience.

Nous avons vu qu'il importait de réagir devant les lacunes constatées dans la formation du traducteur. À cette fin, je propose que l'on aborde l'enseignement de la traduction dans un tout autre esprit, d'un point de vue repensé et intégré, en fonction des véritables besoins des étudiants, qui ne sont pas nécessairement ceux que l'on croit traditionnellement. Nous avons également vu la finalité d'une méthode d'enseignement de la traduction par l'interprétation du texte et sa justification, ainsi que le cheminement au terme duquel j'en suis arrivé à cette conclusion et les fondements théoriques d'une telle méthode. Il reste maintenant à voir comment de la théorie passer à la pratique.

\section{LES BASES D'UNE MÉTHODE GÉNÉRALE D'ENSEIGNEMENT DE LA TRADUCTION}

Si nous partons du constat évoqué au début de mon propos, soit des lacunes avérées dans la formation de nos étudiants (qui ne sont pas, loin de là, toutes imputables à l'université), toute méthode de traduction repensée devrait viser au moins les trois objectifs suivants:

- développer la capacité d'autonomie des étudiants;

- étendre leur culture générale;

- renforcer leurs connaissances linguistiques.

Je crains ici de décevoir le lecteur: je n'ai pas de recette magique à proposer, de panacée à tous nos maux. Il serait d'ailleurs présomptueux et fallacieux de ma part de laisser entendre que l'on peut facilement et simplement résoudre de tels problèmes. Tout au plus peut-on espérer arriver à en réduire l'impact, à en atténuer les effets. Comme je le disais voici un instant, la traduction est un état d'esprit, une attitude devant les langues, les cultures et, finalement, la vie. La traduction est existentielle par définition. Aussi est-il préférable, avant de songer à établir une méthode - mot qui laisse croire erronément qu'il y a une façon ou «manière» de traduire, quand on sait que la traduction est, à l'image de la vie, multiple, protéiforme -, de penser une pédagogie de la traduction, c'est-à-dire de s'interroger d'abord sur son «esprit» et sur les conséquences de toute 
orientation fixée, le plus souvent, de façon arbitraire. Car, ne l'oublions pas, nous ne formons pas seulement des traducteurs, nous avons charge de consciences. Préparer des traducteurs à l'exercice d'un métier difficile, c'est bien et louable. Mais préparer des jeunes gens à la vie professionnelle, fût-ce par le biais de la traduction, et à la vie tout court, c'est à la fois louable et nécessaire. Telle est, ou devrait être, la véritable fonction de l'université. Telle devrait être celle d'un programme de formation des traducteurs.

Plus concrètement, une méthode d'enseignement de la traduction inspirée des préoccupations que j'ai exposées devrait présenter suffisamment de souplesse pour répondre à des besoins très divers. Je veux dire par là qu'elle devrait toujours prévoir deux volets dans l'apprentissage, soit, pour reprendre un vocabulaire bien établi dans certaines disciplines olympiques, des exercices «imposés» et des exercices «libres». Un panachage des deux s'impose. On ne saurait appliquer toutefois les principes des seconds avant d'avoir inculqué ceux des premiers, sans courir le risque de vouloir mettre la charrue devant les bœufs et de faire de nos futurs traducteurs des êtres tronqués, amputés des bases solides à partir desquelles construire l'édifice de leur carrière.

\section{DES IMPOSÉS}

Les premiers sont destinés à développer des réflexes quasi automatiques chez le traducteur, en faisant intervenir la mémoire et la technique pures. Par ce moyen, il est possible d'élever le degré d'autonomie du traducteur, par exemple en lui montrant que l'acquisition systématique de vocabulaire est un acte de libération (en particulier vis-à-vis des dictionnaires, unilingues comme bilingues, dont la consultation irrésistible et effrénée trahit un manque de confiance évident en ses propres capacités) autant qu'un acte d'affirmation et de confiance en soi. Son autonomie s'exercera également sur le plan de la connaissance des langues (au moins passive, s'entend, pour un traducteur; active et passive, pour un interprète) et de la rapidité d'exécution. Pour cela, la consolidation des connaissances linguistiques et la maîtrise progressive de la vitesse de traduction impliquent une double responsabilité, de la part du professeur comme de l'étudiant. C'est, entre autres, par la lecture - mais effectuée dans et à certaines conditions - et par la pratique que le traducteur parviendra à une meilleure connaissance des unes et à une plus grande aisance dans la maitrise de l'autre.

C'est ainsi, par exemple, que j'entraîne mes étudiants avancés à acquérir cette aisance que confêre une pratique répétée de la traduction rapide, sans aucun outil documentaire susceptible de les ralentir dans leur action, effectuée en classe, en temps limité, sur des textes de longueur et de difficulté variables. Avec la pratique et le temps - dans la mesure où l'on en dispose... -, ce genre d'exercice est des plus profitables.

Quant à la «culture générale», il va de soi qu'elle ne s'acquiert pas sans un désir sincère et réciproque de la diffuser et de la recevoir. Le professeur ne peut agir ici que comme conseiller et orienteur. Il a pour obligation principale de proposer une méthode d'acquisition ou de renforcement de cette culture et d'en vérifier les acquis. Mais, encore une fois, l'étudiant ne doit pas s'attendre, ici non plus, à faire l'économie de l'effort ingrat et fastidieux que cet apprentissage exige s'il veut parvenir à un résultat satisfaisant. C'est par la présentation personnelle de la matière, fondée sur une connaissance sûre et synthétique du sujet, que le professeur fera sans doute le mieux passer le message premier. Cela toutefois ne saurait suffire, et il appartient à l'étudiant, sur les bases d'une méthode individuelle élaborée en commun selon ses besoins particuliers, de poursuivre l'entreprise amorcée en classe jusqu'à sa conclusion normale. Mais je déborde du cadre des «imposés» et suis déjà dans les «libres». 
AUX LIBRES

Je prendrai une image pour mieux faire comprendre ce que le concept d'exercices imposés et libres recouvre. Imał̣inez qu'au lieu d'être un traducteur vous êtes un musicien de renom. Vous vous produisez en concert chaque soir ou presque. Vous êtes au sommet de votre art. Et pourtant, avant d'interpréter devant un public de mélomanes les pièces que vous savez jouer par cœur, les yeux fermés, tant vous les avez répétées depuis des mois, vous avez encore passé deux heures ce matin à faire des gammes, à vous exercer, tel un débutant appliqué et consciencieux. Est-ce la triste rançon d'une gloire éphémère? Pas du tout! C'est le lot de tout artiste, fût-il le plus grand. Même si un traducteur peut éprouver quelque peine à voir en Arthur Rubinstein un exemple à suivre, cette étape technique, inlassablement recommencée, dans le parcours d'un grand artiste $n$ 'en est pas moins la condition sine qua non de son succès.

Le traducteur ne fait pas exception à la règle: il faut d'abord faire des gammes des exercices imposés - avant de passer aux figures libres. Par là, j'entends donner libre cours à son imagination et à son inspiration, bref, à son style. Nous verrons tout à l'heure un exemple d'exercices imposés, tels que je les pratique en classe, bien que, encore une fois, de façon intégrée, globale, en fonction des besoins pédagogiques et des objectifs du cours. C'est que le style auquel je fais allusion doit, avant de pouvoir s'exprimer librement et sainement, avoir été travaillé («Vingt fois sur le métier...»), canalisé et parfois même bridé, si l'on veut qu'il rende son plein potentiel. Pour reprendre la parabole du musicien, il faut apprendre à lire les notes sur la portée avant de devenir compositeur et de les y placer au gré de sa fantaisie, mais toujours en fonction d'un support: la portée - le texte, pour le traducteur.

Avant d'en terminer avec mon exposé et de présenter la formule d'exercices imposés que je préconise, je voudrais m'expliquer sur ce que j'entends par méthode générale.

\section{LE DISCOURS GÉNÉRAL DE LA MÉTHODE}

Je n'entrerai pas dans la controverse sur le caractère général ou spécialisé d'un texte qui alimente le débat entre théoriciens. Et de toute façon, ce n'est pas le sens auquel je pense lorsque je parle d'une méthode générale d'enseignement de la traduction, c'est-à-dire applicable à tous les types de textes : technique, commercial, juridique, scientifique, médical, littéraire - ce qui comprend la poésie - etc.

Nous savons depuis longtemps (Vinay et Darbelnet, 1958) que l'on ne traduit bien que ce que l'on comprend - ou ressent? - parfaitement. Chargé d'enseigner une forme avancée et moderne de la version «générale» depuis quelque 15 ans, tantôt appelée «Matières d'actualité» ici, tantôt «Questions d'actualité» là, j'ai pu réfléchir mûrement au problème que pose la connaissance d'une matière dans laquelle l'étudiant n'a pas reçu de formation: le droit pour un scientifique ; l'informatique ou le technique pour un littéraire; la biologie ou la génétique pour un sociologue; etc.

\section{LA CULTURE GÉNÉRALE DU TRADUCTEUR}

Sans pour autant prétendre apporter une réponse définitive ou une solution universelle sur ce point, je pense être au moins en mesure d'apporter quelques éléments de réflexion, voire de solution possible. Par culture générale j'entends une connaissance suffisante des principes généraux d'un domaine. Nul n'attend d'un étudiant en traduction que ses connaissances, en histoire, par exemple, concurrencent celles d'un Fernand Braudel; ni que ses connaissances en économie rivalisent avec celles de Paul Samuelson! Mais on peut attendre d'un étudiant généraliste qu'il sache LIRE le texte qu'il va traduire, autrement dit qu'il le comprenne suffisamment pour le traduire correctement. 
D'un généraliste, j'attendrai qu'il saisisse au moins le sens de surface d'un texte; d'un étudiant spécialisé, qu'il en manifeste une compréhension profonde. Dans le premier cas, l'étudiant doit connaître les notions de base de la matière de telle manière qu'il se sente à l'aise dans son traitement. Ne pas comprendre le principe de supply-side economics (l'économie de l'offre, par opposition à une politique économique fondée sur la demande) de l'Administration Reagan, par exemple, causera bien des déboires à un apprenti économiste. Le spécialiste, lui, sera au fait des enjeux économiques et politiques d'un tel choix et saisira la portée du débat théorique opposant les partisans de l'offre à ceux de la demande, les keynésiens aux tenants de l'école de Chicago.

L'étudiant qui, placé devant le mot debt, traduira dans tous les cas par «dette» ignore la différence entre une dette (Econ.) et une créance (Jur.). Même chose pour celui qui traduit systématiquement le terme law par «loi», alors que le sens le plus fréquent, et de très loin, est celui de «droit» (système) ou «règle de droit/juridique». Je pourrais multiplier ce genre d'exemples à plaisir. Ils n'ont d'autre but que de faire comprendre que, pour traduire dans un domaine $\mathbf{x}$, l'étudiant doit adopter le système d'interprétation propre à celui-ci, se couler dans la mentalité du physicien, du juriste, de l'économiste, du sociologue, du technicien, du biologiste ou... du poète. En d'autres termes, il doit penser comme eux. Pour l'y amener, le professeur de traduction doit adopter successivement ou, parfois, concurremment - au sein d'un même texte, ce qui est fréquent -, la démarche du spécialiste qu'il prétend représenter. Je n'irai pas jusqu'à dire que la chose est facile, du moins est-elle faisable.

C'est par cette voie que le futur traducteur accédera à la culture de son temps; c'est en l'éveillant aux grands problèmes contemporains de société, aux événements d'importance nationale et internationale, en piquant sa curiosité et en faisant appel à son intelligence autant qu'à sa mémoire; c'est en développant sa capacité de réflexion et d'interrogation que l'on parviendra à former des traducteurs aptes à répondre non seulement aux besoins de la société dans laquelle ils vivent, mais aussi aux leurs propres. En somme, des êtres à l'écoute de leur temps et vivant au rythme de sa respiration.

Avant d'en arriver là, l'apprenti traducteur devra maîtriser les techniques de base. Il importe donc d'apprendre à l'étudiant à travailler.

\section{UNE FORMULE D'TNTERPRÉTATION DU TEXTE}

Une des rechniques que j'utilise couramment est celle des cinq niveaux d'interprétation du texte. Elle est loin d'être exhaustive mais suffit pour appréhender les éléments essentiels à la compréhension globale d'un texte donné, quel qu'en soit le domaine.

Ces cinq niveaux sont les suivants:

- sémantique

- grammatical

- syntaxique

- terminologique (ou lexical)

- stylistique.

En schématisant très grossièrement, je dirai que cette formule reprend, sous une forme différente et adaptée aux besoins des traducteurs, le schéma linguistique traditionnel voulant que l'opération de traduction parte du sens pour aller vers la forme.

1. Le sens. Au commencement était le sens, pourrait-on dire. En effet, tout commence par là, et le professeur doit s'assurer que la saisie des significations véhiculées par chaque unité de sens (ou mot) est bien comprise. On peut parler ici de connaissances linguistiques nécessaires et suffisantes pour saisir le sens, même superficiellement, d'un énoncé.

2. La grammaire. L'étape de la grammaire, pour aussi traditionnelle qu'elle puisse paraître, est néanmoins essentielle; en fait, il s'agira de faire de la grammaire comparée 
ou «contrastive», d'analyser ce qui est jugé obligatoire (Hagège, 1985: 241); par exemple, les flexions et désinences, les temps et les modes à utiliser.

3. La syntaxe. L'analyse grammaticale précède celle de la «syntaxe», qui peut être soit «formelle» (analyse des relations entre les divers constituants), soit sémantique (par ex., l'ordre des mots est porteur de sens différents) selon l'orientation que le pédagogue voudra imprimer à son enseignement.

4. La terminologie. Le quatrième niveau d'analyse interprétative est celui de la terminologie (ou du lexique). Par terminologie il faut entendre ici l'aspect notionnel des termes, ce qu'ils recouvrent: les mots et les choses. Cette étape, pour un traducteur, est très importante en ce sens qu'elle permet de travailler à divers paliers de la connaissance d'un sujet, du plus simple au plus complexe (cf. le principe cardinal de la «famille» de mots; par ex., en partant du terme «simple» contrat/contract, remonter jusqu'à la théorie des obligations en droit comparé).

5. La stylistique. Enfin, le niveau stylistique. Il est situé en dernière place pour les raisons invoquées tout à l'heure, ce qui ne veut pas dire qu'il représente nécessairement la dernière roue de la charrette dans l'ordre des préoccupations du traducteur! La stylistique - pour nous : comparée - a pour objet les effets, les niveaux de langue et les divers choix possibles entre des solutions, des manières de dire parfois très proches à l'arrivée (cf. Molière et son vers célèbre: «Marquise, d'amour vos beaux yeux mourir me font» ou «D'amour, Marquise, vos beaux yeux ...», etc.). Bref, le calcul des «possibles».

$\mathrm{Au}$ cours d'une seule et même opération interprétative, il est donc possible de parcourir les diverses avenues qu'emprunte un énoncé, depuis la compréhension brute de son sens jusqu'à ses moyens d'expression, en passant par les fonctions qu'assument ses divers constituants et les notions qu'ils recouvrent. Selon le type d'objectifs qu'il vise, le pédagogue insistera sur tel ou tel aspect en particulier. Par exemple: 1,2 et 3;3, 4 et 5 .

Par cet exercice intégré (les «imposés»), le professeur de traduction devrait être à même de faire le tour de toute question - d'où le postulat de la méthode générale, tous domaines confondus - et de préparer le futur traducteur à remplir ses obligations futures, sur le double plan de la forme et du contenu.

\section{CONCLUSION}

Je conclurai sur la remarque suivante: si la traduction est bien, comme le prétend Isaac Bashevis Singer, «l'essence même de la civilisation», la pédagogie de la traduction que je préconise, appliquée comme méthode d'interprétation du texte, n'a d'autre ambition que de tenter de faire justice à la richesse et à la diversité de notre monde et de sa civilisation, et de faire du traducteur son héraut.

* Je dédie le présent travail à la mémoire de ma collègue Irène Spilka.

\section{BIBLIOGRAPHIE}

BOUTON, Charles (1984) : «Le cerveau du traducteur», Meta, 29-1, numéro spécial : Cerveau, langage et traduction, Montréal, Les Presses de l'Université de Montréal, p. 44. CHANGEUX, Jean-Pierre (1983) : L' homme neuronal, Paris, Fayard.

CHARAUDEAU, Patrick (1983) : Langage et discours. Éléments de sémiolinguistique, Paris, Hachette.

CHOMSKY, Noam (1965): Aspects of the Theory of Syntax, Cambridge, MIT Press.

CHOMSKY, Noam (1980): Essai sur la forme et le sens, Paris, Seuil.

DAVID, Aurel (1980): Le bon partage des biens, Ottawa, Éditions de l'Université d'Ottawa.

DELISLE, Jean (1980): L'analyse du discours comme méthode de traduction, Ottawa, Éditions de l'Université d'Ottawa.

HAGËE, Claude (1982) : La structure des langues, Que sais -je ?, Paris, PUF.

HAGEGE, Claude (1985) : L'homme de paroles, Paris, Fayard.

JACOB, François (1970): La logique du vivant : une histoire de l'hérédité, Paris, Gallimard. 
JACOB, François (1981): Le jeu des possibles. Essai sur la diversité du vivant, Paris, Fayard.

JACQUARD, Albert (1978): Éloge de la différence : la génétique et les hommes, Paris, Seuil.

JACQUARD, Albert (1982): Au péril de la science?, Paris, Seuil.

JACQUARD, Albert (1986): L'héritage de la liberté. De l'animalité à l'animalitude, Paris, Seuil.

LARKIN, Jill et al. (1980): «Expert and novice performance in solving Physics Problems», Science, 208, p. 1335.

LE DORZE, G. et J.-L. NESPOULOUS (1984) : «Modèles psycholinguistiques et leur application à l'étude de l'aphasie et de la traduction», Meta, 29-1, Montréal, Les Presses de l'Université de Montréal.

NESPOULOUS, J.-L. (1984) : «En guise d'introduction... Neurolinguistique, psycholinguistique et traduction», Meta, ibid., p. 8.

PARADIS, Michel (1984): «Aphasie et traduction», Meta, ibid., p. 57.

PARADIS, M. et A. R. LECOURS (1984) : «L'aphasie chez les bilingues et les polyglottes», l'Aphasie, A. R. LECOURS, F. LHERMITTE et al. (réd.), Paris, Flammarion, p. 605.

PIAGET, Jean (1970) : «Mémoire et intelligence», la Mémoire, B. Bovet et al., Paris, PUF.

POLANYI, Michael (1967): «Sense-Giving and Sen'se-Reading», Philosophy, XLII-162, p. 301.

PRIGOGINE, IIya et Isabelle STENGERS (1979): La nouvelle alliance, Paris, Gallimard.

SEARLE, John (1986): Du cerveau au savoir, Paris, Hermann.

THOMAS, Raymond (1982): Évaluation de l'information en langues naturelles, thèse de doctorat d'État Université de Caen, U.E.R. des langues vivantes étrangères (non publiée).

VINAY, J.-P. et J. DARBELNET (1958) : Stylistique comparée du français et de l'anglais, Paris, Didier.

WALDROP, M. Mitchell (1984) : «The Necessity of K

nowledge», Science, 223-4642, p. 1279. 